\title{
Where sexes collide
}

The males and females of many species have different interests at heart; this is reflected in their appearance, behaviour and, ultimately, in their DNA, in which genes are evolutionarily torn as to whether to benefit one sex or the other. A study in fruitflies has mapped the loci at which this genomic tug-of-war takes place and shows that such genes abound on the $\mathrm{X}$ chromosome.

Innocenti and Morrow focused on a common sexually antagonistic trait: the inverse correlation between the reproductive fitness of males and females in Drosophila melanogaster. In a competitive mating experiment on 100 fly lines, the authors confirmed that, indeed, lines with high-fitness males contained low-fitness females, and vice versa. To identify the genes that underlie this conflict, the authors ran transcriptomics experiments on 15 lines of flies, split equally into three classes according to whether fitness was the same across the sexes or whether the fitness in one sex was higher than in the other.

Most (90\%) of the $\sim 19,000$ fly transcripts were differentially expressed between males and females. However, a surprisingly small proportion of these was associated with the reproductive fitness of males or females. In that fraction there were 1,500 transcripts (corresponding to 1,300 genes) that benefited one sex while harming the fitness of the other - this represents only $8.5 \%$ of sex-biased transcripts and so warns against using sexbiased expression as an indicator of sexually antagonistic function.

The loci were enriched in 68 genomic regions and were especially enriched on the X chromosome. This is as theory predicts: male fruitflies have only one $\mathrm{X}$ chromosome, so an $\mathrm{X}$-linked recessive gene that benefits males can be phenotypically silenced in XX females.

This is the first study in which sexually antagonistic genes have been mapped in any species. Information about the number, nature and location of these loci will be welcomed by researchers of this taxonomically widespread phenomenon.

Tanita Casci
ORIGINAL RESEARCH PAPER Innocenti, P. \& Morrow, E. H. The sexually antagonistic genes of Drosophila melanogaster. PLoS Biol. 8, e1000335 (2010)

FURTHER READING Williams, T. M. \& Carroll, S. B. Genetic and molecular insights into the development and evolution of sexual dimorphism. Nature Rev. Genet. 10, 797-804 (2009)

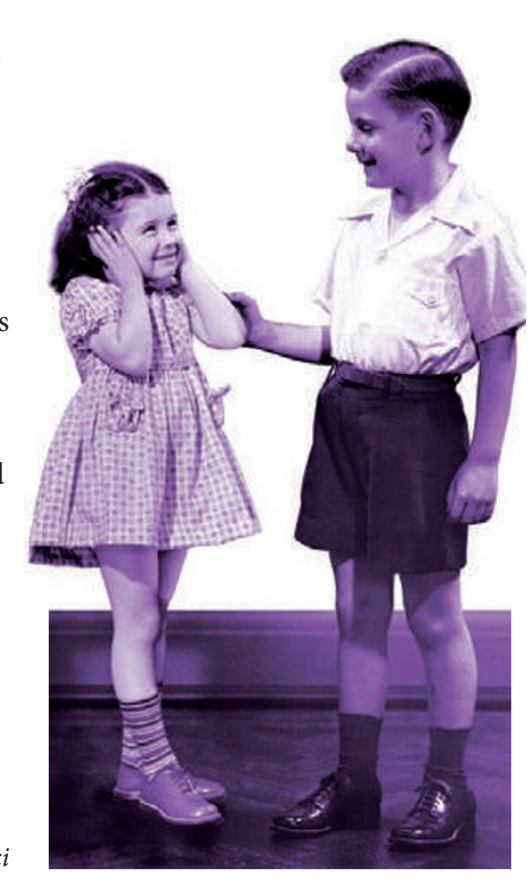

